\title{
Parameters of the energy spectrum for holes in $\mathrm{CuInSe}_{2}$
}

\author{
P.M. Gorley ${ }^{1}$, I.V. Prokopenko ${ }^{2}$, O.O. Galochkina ${ }^{1}$, P.P. Horley ${ }^{1,3}$, Yu.V. Vorobiev ${ }^{4}$, J. González-Hernández ${ }^{5}$ \\ ${ }^{I}$ Yu. Fedkovych Chernivtsi National University, 2, Kotsyubynsky str., 58012 Chernivtsi, Ukraine, \\ phone: +38(03722) 46-877, e-mail:semicon-dpt@,chnu.edu.ua \\ ${ }^{2}$ V. Lashkaryov Institute of Semiconductor Physics, NAS of Ukraine, 41, prospect Nauky, 03028 Kyiv, Ukraine \\ ${ }^{3}$ Centro de Física das Interacções Fundamentais (CFIF), Instituto Superior Técnico, \\ Avenida Rovisco Pais, 1049-001 Lisboa, Portugal \\ ${ }^{4}$ CINVESTAV-IPN Unidad Querétaro, Libramiento Norponiente 2000, Fracc. Real Juriquilla, \\ 76230 Querétaro, México \\ ${ }^{5}$ CIMAV, Miguel de Cervantes 120, Complejo Industrial Chihuahua, 31109 Chihuahua, México
}

\begin{abstract}
This paper reports the coefficients $C_{A, B}$ for the $k$-linear term in dispersion relation $E(\mathrm{k})$ for holes of the upper valence bands $\Gamma_{6}^{-}$and $\Gamma_{7}^{+}$in $\mathrm{p}-\mathrm{CuInSe} \mathrm{e}_{2}$ crystals. We also obtained the tensor components for the carrier effective masses $m_{\perp, \|}^{A, B, C}$ in all three valence sub-bands of the model semiconductor. It was shown that the energy spectrum parameters for holes in $\mathrm{CuInSe}_{2}$ allow successful explanation for the anisotropy of tensor components describing the interband light absorption coefficient and the published data for the temperature variation of the Hall coefficient, total Hall mobility and thermal voltage within the temperature range $100 \mathrm{~K} \leq T \leq 350 \mathrm{~K}$.
\end{abstract}

Keywords: chalcopyrite structure, $\mathrm{CuInSe}_{2}$, non-parabolic dispersion relation, components of the effective mass tensor, kinetic coefficient, light absorption coefficient.

Manuscript received 28.04.09; accepted for publication 00.00.09; published online 00.00.09.

\section{Introduction}

Direct-band chalcopyrite compound $\mathrm{CuInSe}_{2}$ belongs to $\mathrm{A}^{\mathrm{I}} \mathrm{B}^{\mathrm{II}} \mathrm{C}^{\mathrm{VI}}{ }_{2}$ semiconductors that are considered as analogs to $\mathrm{A}^{\mathrm{II}} \mathrm{B}^{\mathrm{VI}}$ binary systems. Due to their high absorption coefficient [1] (about $(3 \ldots 6) \cdot 10^{7} \mathrm{~m}^{-1}$ ) these materials are very promising for the efficient photovoltaic device applications [2]. In general, $\mathrm{CuInSe}_{2}$ crystals are studied in deeper detail comparing with the other $\mathrm{A}^{\mathrm{I}} \mathrm{B}^{\mathrm{II}} \mathrm{C}_{2}{ }_{2}$ compounds (e.g., [3-5]). However, the exact nature of some of their properties is not clearly revealed yet. In particular, it concerns the fundamental parameters characterizing the valence band structure. According to the results of the theoretical calculations $[6,7]$, the band structure of the bulk $\mathrm{A}^{\mathrm{I}} \mathrm{B}^{\mathrm{II}} \mathrm{C}^{\mathrm{VI}}{ }_{2}$ semiconductors in the center of the Brillouin zone obeys anisotropic nonparabolic dispersion relation $E(\mathbf{k})$ including a k-linear term. To the best of the authors' knowledge, the numerical values of the coefficients $\mathrm{C}$ describing the aforementioned term are not determined yet for $\mathrm{p}$ $\mathrm{CuInSe}_{2}$ crystals, while they are known for the $\mathrm{A}^{\mathrm{II}} \mathrm{B}^{\mathrm{VI}}$ materials p-CdS, p-CdSe and p-ZnO. Moreover, only three out of six components of the hole effective masses for $\mathrm{p}-\mathrm{CuInSe} \mathrm{Se}_{2}$ were determined so far from the optical studies [8]: those for spin-split band holes $\left(m_{s h} / m_{0} \approx 0.085\right)$, heavy $\left(m_{h h} / m_{0} \approx 0.71\right)$ and light holes $\left(m_{h l} / m_{0} \approx 0.092\right)$.

Our previous research [9] considered nonparabolicity of the dispersion relation $E(\mathbf{k})$ for the holes in chalcopyrite-structure crystals under non-degenerate statistics of the electron gas, deriving the analytical expressions allowing to calculate the temperature and concentration dependences of tensor components for the thermal voltage, Hall coefficient and carrier mobility. Carrier scattering by crystalline lattice defects was considered in the approximation of time relaxation. For the upper valence sub-bands $\Gamma_{6}^{-}$and $\Gamma_{7}^{+}$of $\mathrm{p}-\mathrm{CuInSe}{ }_{2}$, we estimated the coefficients $C_{\Gamma_{6}^{-}, \Gamma_{7}^{+}}$characterizing the contribution of $\mathbf{k}$-linear term into $E(\mathbf{k})$. The results of further calculations agreed well with the experimental temperature dependences of the Hall coefficient and total Hall carrier mobility.

To improve the approach used in [9], we suggest also to account for the symmetry relations binding the tensor components of the inverse effective masses for 
the holes $\left(m_{\Gamma_{6}^{-}, \Gamma_{7}^{+}, \Gamma_{7}}\right)^{-1}$ in chalcopyrite (wurtzite) crystals [10-12]. This methodological improvement would change the values of $C_{\Gamma_{6}^{-}, \Gamma_{7}^{+}}$obtained in [9], leading to more exact results concerning the influence of $k$-linear term of $E(\mathbf{k})$ on the values and temperature behavior of the kinetic coefficients in p-CuInSe ${ }_{2}$. Moreover, proper accounting of the symmetry for the components $\left(m_{\Gamma_{6}^{-}, \Gamma_{7}^{+}, \Gamma_{7}}\right)^{-1}$ allows to get an accurate magnitude estimation for all the inverse effective mass components describing the holes in three valence subbands of the material studied. Fitting the theoretical calculations to the experimental data on temperature dependences for the Hall coefficient, Hall mobility and thermal voltage in $\mathrm{p}$-CuInSe $\mathrm{C}_{2}$ crystals allowed to improve precision in determining $C_{\Gamma_{6}^{-}, \Gamma_{7}^{+}}$coefficients.

We have also shown that non-parabolicity contribution to $E(\mathbf{k})$ in the total Hall mobility decreased from about $50 \%(T=100 \mathrm{~K})$ to $17 \%(T=300 \mathrm{~K})$, enhancing the previously reported data of $\approx 20 \%$ and $\approx 10 \%$, respectively [9].

\section{Theory}

Schematic depiction of the valence band diagram $[4,13]$ for $\mathrm{p}-\mathrm{CuInSe} \mathrm{S}_{2}$ around the center of the Brillouin zone is shown in Fig. 1.

The dispersion relation for the carriers populating the valence bands $\Gamma_{6}^{-}, \Gamma_{7}^{+}$and $\Gamma_{7}$ (taking into account spin-orbital interaction) can be written in the following form [7]:

$$
\begin{aligned}
& E_{\Gamma_{6}^{-}, \Gamma_{7}^{+}}(\vec{k})=E_{0}^{A, B}+\frac{\hbar^{2}}{2 m_{\perp}^{A, B}} \times \\
& \times\left(k_{\perp} \mp m_{\perp}^{A, B} \cdot C_{A, B} / \hbar^{2}\right)^{2}+\frac{\hbar^{2} k_{\|}^{2}}{2 m_{\|}^{A, B}},
\end{aligned}
$$

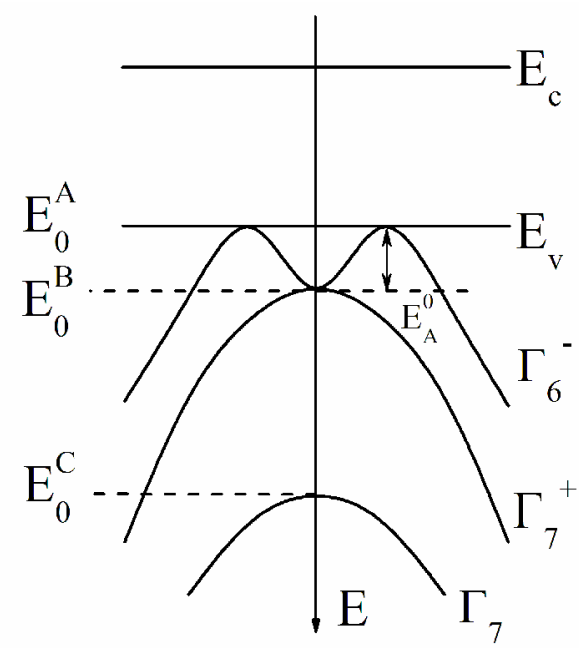

Fig. 1. Band diagram for $\mathrm{p}-\mathrm{CuInSe} \mathrm{C}_{2}$; the designations are explained in the text.
$E_{\Gamma_{7}}(\vec{k})=E_{0}^{C}+\frac{\hbar^{2}}{2}\left(\frac{k_{\perp}^{2}}{m_{\perp}^{C}}+\frac{k_{\|}^{2}}{m_{\|}^{C}}\right)$,

with transversal $k_{\perp}=\left(k_{x}^{2}+k_{y}^{2}\right)^{1 / 2}$ and longitudinal $k_{\|}=k_{z}$ components of the wave vector $\mathbf{k}$ for $m_{\perp}^{A, B, C}$ and $m_{\|}^{A, B, C}$ effective mass tensors describing the holes in the subbands $\Gamma_{6}^{-}$(index A), $\Gamma_{7}^{+}$(index B) and $\Gamma_{7}$ (index C) regarding the high-symmetry axis of the crystal. The coefficients $C_{A, B}$ characterize the deviation from parabolicity in the dispersion relation $E(\mathbf{k})$ for the corresponding valence sub-bands. $E_{0}^{A, B, C}$ in (1) and (2) describe the position of their extrema.

As the dispersion relation (1) is characteristic for the semiconductors with chalcopyrite or wurtzite structure of [14-17], it is natural to assume that the components of the effective mass tensors for $\mathrm{p}-\mathrm{CuInSe} \mathrm{C}_{2}$ would also satisfy the relations obtained for wurtzite crystals $[11,12]$ :

$\frac{m}{m_{\|}^{B, C}}-\frac{m}{m_{\|}^{A}}=\Delta\left(\frac{m}{m_{\perp}^{B, C}}-\frac{m}{m_{\perp}^{A}}\right)$,

$\frac{m}{m_{\perp}^{A}}-\frac{m}{m_{\|}^{A}}=\frac{(g-r)\left(2+\Delta_{1}\right)}{1+C_{2}+C_{3}}$,

$\frac{m}{m_{\|, \perp}^{B}}-\frac{m}{m_{\|, \perp}^{A}}=C_{1}\left(\frac{m}{m_{\|, \perp}^{C}}-\frac{m}{m_{\|, \perp}^{A}}\right)$,

$\frac{m}{m_{\perp}^{C}}-\frac{m}{m_{\|}^{C}}=C_{2}\left(\frac{m}{m_{\perp}^{A}}-\frac{m}{m_{\|}^{A}}\right)$,

$\frac{m}{m_{\perp}^{B}}-\frac{m}{m_{\|}^{B}}=C_{3}\left(\frac{m}{m_{\perp}^{A}}-\frac{m}{m_{\|}^{A}}\right)$.

Here $g, r, s, t$ denote $\hbar^{2} / 2 m$-normalized ( $\hbar$ is Planck's constant and $m$ is the free electron mass) matrix elements for the interband interaction operators [12], with adjustment parameters

$$
\begin{aligned}
\Delta= & \frac{t-r}{s-g}, \Delta_{1}=\frac{s-t}{g-r}, C_{2}=\left(1-q^{2}\right) \cdot\left(C_{1}+\Delta_{1}\right), \\
C_{3}= & \left(1-q^{2}\right) \cdot\left(1+\Delta_{1} C_{1}\right) . \\
& \text { The coefficient } \\
C_{1}= & q^{2} /\left(1-q^{2}\right)
\end{aligned}
$$

with

$$
q^{2}=\frac{1}{2}\left[1-\frac{x}{\sqrt{x^{2}+8 / 9}}\right]
$$

depends on the energy split $x=\Delta_{c r} / \Delta_{s o}-1 / 3$ caused by the crystalline field $\Delta_{c r}$ and spin-orbital interaction $\Delta_{s o}[10]$. It is important that the $m c$-normalized ( $m$ is the electron mass and $c$ is the speed of light) matrix elements for the optical transitions from $\mathrm{B} / \mathrm{C}$ valence 
sub-bands to the conduction band [10] also depends on $q^{2}$

$M_{\|}^{B}=2 M_{\perp}^{C}=q^{2}, M_{\|}^{C}=2 M_{\perp}^{B}=1-q^{2}$,

while for the A-band $M_{\|}^{A}=0, M_{\perp}^{A}=0.5$.

The experimental values $\Delta_{c r}=0.006 \mathrm{eV}$ and $\Delta_{s o}=0.233 \mathrm{eV}$ [18] lead to $C_{1} \approx 1.9>1$, proving that for $\mathrm{p}-\mathrm{CuInSe} \mathrm{S}_{2}$ (and also CdSe, $\mathrm{ZnSe}$ ) the components of the effective mass tensor for holes in the valence subbands would satisfy the condition [12]:

$m_{\|}^{B}<m_{\|}^{C}<m_{\|}^{A}, m_{\perp}^{A}<m_{\perp}^{C}<m_{\perp}^{B}$.

It is important that for $C_{1}<1$ (which is valid for $\mathrm{CdS}, \mathrm{ZnS}, \mathrm{ZnO}$ and $\mathrm{GaN}$ ), the components of symmetry for the effective mass tensor are [10-12]

$m_{\|}^{C}<m_{\|}^{B}<m_{\|}^{A}, m_{\perp}^{A}<m_{\perp}^{B}<m_{\perp}^{C}$.

Comparison of (8) and (9) shows that changing $C_{1}>1$ for $C_{1}<1$ will result in swapping of the valence sub-bands $\mathrm{B}$ and $\mathrm{C}$.

Formulas (5) and (7) yield a proportion $C_{1}=M_{\|}^{B} / M_{\|}^{C}=M_{\perp}^{C} / M_{\perp}^{B}$ saying that the coefficient $C_{1}$ depends on the ratio of matrix elements for the optical transitions (with the same polarization) from the bands $\mathrm{B} / \mathrm{C}$ into conduction band. Therefore, one can assume that $C_{1}$ should contribute somehow to the anisotropy of the light absorption coefficient $\alpha_{\|} / \alpha_{\perp}$ (with parallel and perpendicular subscripts denoting light polarization regarding the main optical axis of the crystal).

\section{Results and discussion}

Using the symmetry relations (3) one can show that for the present experimental effective mass values describing the holes of $\mathrm{p}-\mathrm{CuInSe} \mathrm{C}_{2}$ [8] the inequalities (8) would be valid for these two particular cases (within parameters with the precision of experimental measurements):

Case $\mathrm{D}_{1}: \frac{m_{\|}^{A}}{m}=0.091, \frac{m_{\|}^{B}}{m}=0.074, \frac{m_{\|}^{C}}{m}=0.081$,

$\frac{m_{\perp}^{A}}{m}=0.087, \frac{m_{\perp}^{B}}{m}=0.73, \frac{m_{\perp}^{C}}{m}=0.162 ;$

Case $\mathrm{D}_{2}: \frac{m_{\|}^{A}}{m}=0.71, \frac{m_{\|}^{B}}{m}=0.165, \frac{m_{\|}^{C}}{m}=0.260$,

$\frac{m_{\perp}^{A}}{m}=0.078, \frac{m_{\perp}^{B}}{m}=0.092, \frac{m_{\perp}^{C}}{m}=0.085$.

Table 1 presents the anisotropy coefficients for effective masses of holes $K_{m \perp, \|}^{A, B, C}=\frac{m_{\|}^{A, B, C}}{m_{\perp}^{A, B, C}}$. We calculated the data both for $\mathrm{p}-\mathrm{CuInSe} \mathrm{C}_{2}$ crystals and their $\mathrm{A}^{\mathrm{II}} \mathrm{B}^{\mathrm{VI}}$ analogs using the experimental mass values for $\mathrm{CdSe}, \mathrm{CdS}$ [10] and $\mathrm{ZnO}$ [14].
Table 1. Anisotropy coefficients for hole effective masses in p-CuInSe $e_{2}$ and $\mathrm{A}^{\mathrm{II}} \mathrm{B}^{\mathrm{VI}}$ analogs.

\begin{tabular}{|c|c|c|c|c|c|c|}
\hline \multirow{2}{*}{ Material } & \multicolumn{2}{|c|}{$K_{m}^{A}$} & $K_{m}^{B}$ & $K_{m}^{C}$ & $E_{g}, \mathrm{eV}$ & $C_{1}$ \\
\hline \multirow{2}{*}{ p-CIS } & Case C1 & 1.05 & 0.10 & 0.50 & & \multirow{2}{*}{1.90} \\
\cline { 2 - 6 } & Case C2 & 9.10 & 1.79 & 3.06 & $1.010^{[22]}$ & \\
\hline p-CdSe & 2.66 & 0.53 & 0.91 & $1.756^{[23]}$ & 1.65 \\
\hline p-CdS & 4.70 & 0.76 & 0.48 & $2.485^{[23]}$ & 0.73 \\
\hline p-ZnO & \multicolumn{2}{|c|}{5.07} & 3.76 & 0.24 & $3.370^{[23]}$ & 0.035 \\
\hline
\end{tabular}

As one can see, for the case $C_{1}(10)$ with increasing band gap $E_{g}$ (parameter $C_{1}$ decreases) the anisotropy coefficient for effective masses of the holes populating $\mathrm{A}$ and $\mathrm{B}$ valence bands also increases. At the same time, the coefficient for the C-band decreases for all the studied materials except for $\mathrm{CdSe}$. Effective mass components calculated for the case $\mathrm{D}_{2}$ (11) for $\mathrm{CuInSe} \mathrm{S}_{2}$ violate this regularity for $\mathrm{A}$ and $\mathrm{B}$-bands, to the contrary restoring it for the C-band.

To define which of the cases $D_{1}$ or $D_{2}$ is correct for p-CuInSe ${ }_{2}$, we used analytical expressions from [9] to calculate temperature behavior of the tensors describing the specific conductivity, Hall mobility and thermal voltage. To define the fixed $m_{\perp, \|}^{A, B, C}$, the coefficients $C_{A, B}$ in the dispersion relation (1) were considered as parameters and adjusted to achieve the best fitting of the calculated data to the experimental temperature curves for the kinetic coefficients. The numerical values of $C_{A, B}$ for $\mathrm{p}-\mathrm{CuInSe} \mathrm{S}_{2}$ and $\mathrm{A}^{\mathrm{II}} \mathrm{B}^{\mathrm{VI}}$ are given in Table 2.

It is noteworthy that for all the studied compounds we achieved an essentially linear dependence $C_{B}=4.65 \cdot 10^{-2}\left(4.15-E_{g}\right) \mathrm{eV} \cdot \AA \quad$ (Fig. 2, line 2), allowing to assume the linearity of $C_{A}=\psi\left(E_{g}\right)$ as well. To obtain the latter (Fig. 2, line 1) the values of $C_{A}$ for CdSe and CdS should be used in the place of those from [15], which will allow to get the refined coefficients (Table 2). Validity of $C_{A, B}=k_{A, B}\left(E_{0}-E_{g}\right.$ ) dependence for various wurtzite and chalcopyrite crystals makes it possible to estimate the limit values of $C_{A, B}$ coefficients for the case $E_{g} \rightarrow 0$, yielding $C_{A}^{\max }=0.263 \mathrm{eV} \cdot \AA \quad$ and $\quad C_{B}^{\max }=0.193 \mathrm{eV} \cdot \AA$, respectively. On the other hand, for semiconductor with the considered crystalline structure and a bandgap of $E_{g} \geq 4.15 \mathrm{eV}$, the contribution of $\mathbf{k}$-linear term into $E(\mathbf{k})$ should be negligibly small. Due to this fact, for example, the bandgap of $\mathrm{BeO}$ is not determined yet exactly, so that the publication [19] usually mentions the data in a wide range of $7.8-10.6 \mathrm{eV}$. The fact that the linear dependence $C_{A, B}=k_{A, B}\left(E_{0}-E_{g}\right)$ holds for $\mathrm{p}$ $\mathrm{CuInSe}_{2}$ crystals and their $\mathrm{A}^{\mathrm{II}} \mathrm{B}^{\mathrm{VI}}$ analogs suggests that the obtained $C_{A, B}$ coefficients are determined correctly and reliably. 


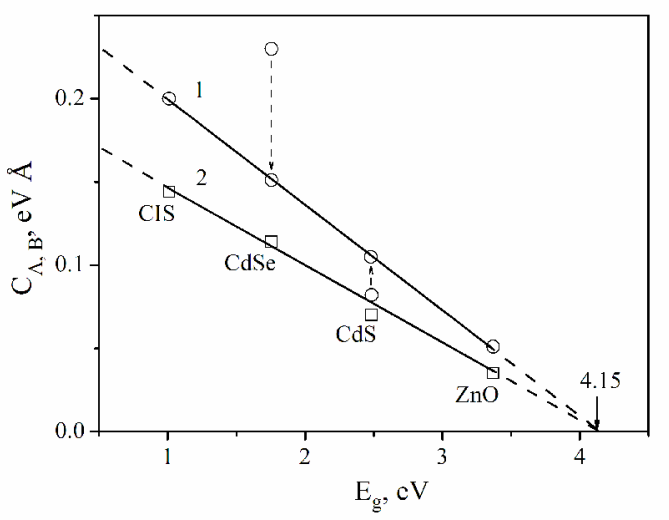

Fig. 2. Dependence of $C_{A}$ (line 1) and $C_{B}$ (line 2) coefficients from (1) on the bandgap of p-CuInSe $e_{2}$ crystals and their $\mathrm{A}^{\mathrm{II}} \mathrm{B}^{\mathrm{VI}}$ analogs. The arrows mark the refined $C_{A}$ for CdSe and $\mathrm{CdS}$, which fits the linear dependence $C_{A}=6.34 \cdot 10^{-2} \cdot\left(4.15-E_{g}\right) \mathrm{eV} \cdot \AA$.

Table 2. Coefficients $C_{A, B}$ for $\mathrm{p}-\mathrm{CuInSe} \mathrm{C}_{2}$ and their $\mathrm{A}^{\mathrm{II}} \mathrm{B}^{\mathrm{VI}}$ analogs $(\mathrm{eV} \cdot \AA ̊ \AA$ units $)$.

\begin{tabular}{|c|c|c|c|c|}
\hline$\frac{\bar{\pi}}{\sum_{\overline{0}}^{\frac{\pi}{\pi}}}$ & 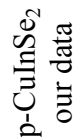 & 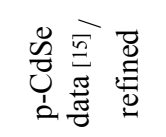 & 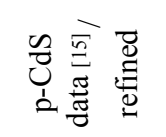 & 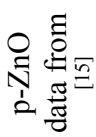 \\
\hline$\overline{C_{A}}$ & 0.200 & $0.230 / 0.150$ & $0.082 / 0.105$ & 0.051 \\
\hline$C_{B}$ & 0.144 & 0.114 & 0.070 & 0.035 \\
\hline
\end{tabular}

Concerning the temperature behavior of kinetic coefficients in $\mathrm{p}-\mathrm{CuInSe} \mathrm{S}_{2}$, our calculations show that the carrier contribution to the valence band $\Gamma_{7}$ is negligibly small in comparison with that of the holes populating $\Gamma_{6}^{-}$and $\Gamma_{7}^{+}$bands. Let us consider the system with a single acceptor level formed by interstitial selenium atoms [20] with the concentration $N_{a} \approx 4.0 \times 10^{18} \mathrm{~cm}^{-3}$ and depth $E_{V}+0.019 \pm 0.002 \mathrm{eV}$ correlating with the data from [21]. We performed calculations for this system by using carrier effective masses calculated for either of $\mathrm{C}_{1}$ and $\mathrm{C}_{2}$ cases (10), (11). The results revealed good agreement between theoretical and experimental [9] results concerning the temperature dependence of the Hall coefficient $R=f(1 / T)$. The calculated thermal voltage degenerates into a scalar value, fitting the experimental data [9] within the precision limits of the experimental measurements.

Solid and dashed curves in Figure 3 present calculation results for the cases $\mathrm{D}_{1}$ and $\mathrm{D}_{2}$, respectively. The curves designated numbers with primes $1^{\prime}, 2^{\prime}$ were obtained for $C_{A, B} \neq 0$. The temperature dependence of the total Hall mobility for holes $u_{\mathrm{H}}(T)$ in p-CuInSe crystals for the different experimental results are shown with circles (data [24]) and triangles (data [25]). Comparing the results presented by curves 1 and $l^{\prime}$ with 2 and $2^{\prime}$, one can see that for the case when nonparabolicity of $E(\mathbf{k})$ is neglected, one obtains the excess

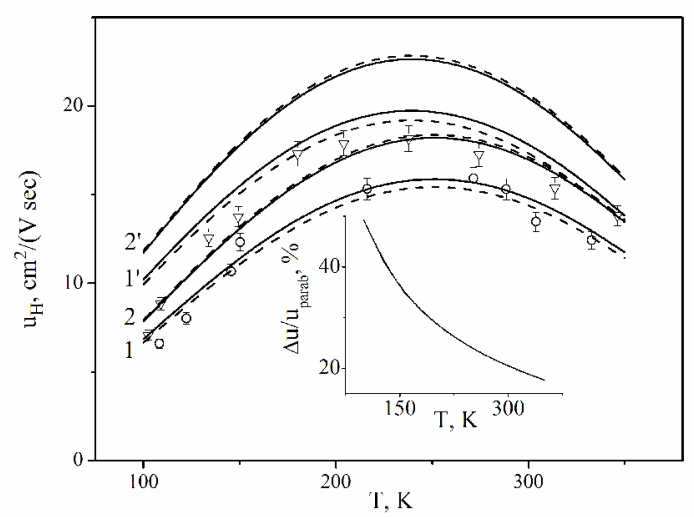

Fig. 3. Temperature dependence of the total Hall mobility for holes in $\mathrm{p}-\mathrm{CuInSe} \mathrm{e}_{2}$. The curve numbering is discussed in the text. Error bars illustrate precision of the experiment.

mobility and markedly different shape of the curve $u_{\mathrm{H}}(T)$. For $C_{A, B} \neq 0$, the calculated and experimental curves for $u_{\mathrm{H}}(T)$ fit each other well for the temperatures $170 \mathrm{~K} \leq T \leq 350 \mathrm{~K}$. The existing mismatch between theory and experiment for $u_{\mathrm{H}}(T)$ in p-CuInSe $\mathrm{C}_{2}$ crystals under $T<150 \mathrm{~K}$ may stem from neglecting the hopping scattering, which becomes quite significant at these temperatures [20]. Under the temperature increase from 100 to $300 \mathrm{~K}$ the contribution of non-parabolicity into the hole mobility was calculated using the masses (10), (11) and the experimental data [24, 25]. We observed a decrease of aforementioned contribution for approximately 33 percents (from $\approx 50 \%$ at $100 \mathrm{~K}$ to $\approx 17 \%$ at $300 \mathrm{~K}$ ), which is illustrated at the inset to Fig. 2 for the parameter $\Delta u / u \equiv\left(u_{\mathrm{H}}\left(C_{A, B} \neq 0\right) /\right.$ $\left.u_{\mathrm{H}}\left(C_{A, B}=0\right)-1\right) \cdot 100 \%$. The resulting difference between the values of the total mobility calculated using $\mathrm{D}_{1}$ and $\mathrm{D}_{2}$ effective hole masses for $\mathrm{p}-\mathrm{CuInSe} \mathrm{I}_{2}$ (the corresponding solid and dashed curves in Fig. 2) are within the experimental precision. Due to this, it is hard to say which set of $m_{\perp, \|}^{A, B, C}\left(\mathrm{D}_{1}\right.$ or $\left.\mathrm{D}_{2}\right)$ is preferable for the description of temperature variation of the kinetic coefficients in $\mathrm{p}-\mathrm{CuInSe}{ }_{2}$.

To solve this problem, we used the experimental spectrum [26] for imaginary part $k_{i}$ of the refraction coefficient $n$ of $\mathrm{CuInSe}_{2}$, measured for different polarizations $i=\perp, \|$ regarding the main crystalline axis and the incident light wave with a wavelength $\lambda$. The knowledge of the latter allowed us to apply the formula [26]

$\alpha_{i}=4 \pi k_{i} / \lambda$

to determine the components of absorption coefficient $\alpha_{i}$. On the other hand [20], the expression for $\alpha_{i}$ at the fundamental absorption edge in semiconductor material can be written as:

$n \cdot \alpha_{i} \cdot \hbar \omega=A_{i} \sqrt{\hbar \omega-E_{g}}$, 
where

$$
A_{i}=\frac{c \cdot m^{3 / 2}}{6 \pi V \varepsilon_{0}} \cdot \frac{e^{2}}{\hbar^{2}} \cdot \sum_{n=A, B, C}\left(2 m_{i r}^{n} / m\right)^{3 / 2}\left(M_{i}^{n}\right)^{2} .
$$

Here $n$ is the carrier concentration, $\hbar \omega$ is the photon energy, $\frac{1}{m_{i r}^{n}}=\frac{1}{m_{i}^{e}}+\frac{1}{m_{i}^{n}}$ describes the $i$-th component of the reduced mass of electron-hole pair ( $m_{i}^{e}$ is the $i$-th component of effective mass tensor for conduction band electrons), $M_{i}^{n}$ are matrix elements for optical transitions (6), $V$ is the volume of the crystal, $e$ is the elementary charge, and $\varepsilon_{0}$ is the absolute dielectric permittivity.

Calculating $\mathrm{D}_{1}$ and $\mathrm{D}_{2}$ effective masses for the holes (Table 1) and using electron effective masses (for $\mathrm{CuInSe}_{2}-m_{\perp} / m=0.10, \quad m_{\|} / m=0.11$ [24], for CdSe - $m_{\perp} / m=0.13, \quad m_{\|} / m=0.14$ [15], for CdS $m_{\perp} / m=0.21, \quad m_{\|} / m=0.22 \quad[15]$ and for $\mathrm{ZnO}-$ $\left.m_{\perp} / m=0.30, \quad m_{\|} / m=0.31 \quad[27]\right), \quad$ we calculated dimensionless variable $A_{\perp, \|}^{*} \equiv \frac{6 \pi V \varepsilon_{0}}{c \cdot m^{3 / 2}} \cdot\left(\frac{e}{\hbar}\right)^{2} \cdot A_{\perp, \|}$ as a function of semiconductor bandgap $E_{g}$ (Fig. 4).

As it can be seen from the figure, the dependence $A_{\perp, \|}^{*}=f\left(E_{g}\right)$ can be quite well described within experimental precision for $m_{\perp, \|}^{A, B, C}, \Delta_{c r}$ and $\Delta_{s o}$ as

$A_{\perp, \|}^{*}=\beta_{\perp, \|} E_{g}^{2} /\left(E_{g}+\gamma_{\perp, \|}\right)$.

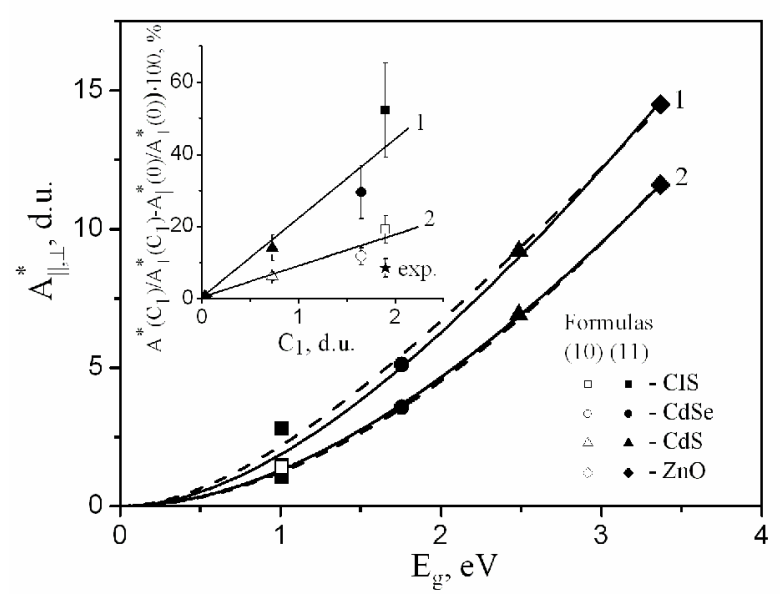

Fig. 4. $A_{\|}^{*}$ (1) and $A_{\perp}^{*}$ (2) coefficients as the function of $E_{g}$ for p-CuInSe $e_{2}$ and its $\mathrm{A}^{\mathrm{II}} \mathrm{B}^{\mathrm{VI}}$ analogs (solid and dashed curves correspond to $\mathrm{D}_{1}$ and $\mathrm{D}_{2}$ hole masses, respectively). The inset shows the ratio $A_{\|}^{*} / A_{\perp}^{*}$ with variation of the parameter $C_{1}$ : line 1 - for the masses (11) and line 2 - for the masses (10). The error bars illustrate dispersion of the theoretical values obtained for the different experimental data. The asterisk denotes the experimental result for $\mathrm{p}-\mathrm{CuInSe} \mathrm{S}_{2}$ [22].
For further calculations, one should use the following values for $\beta_{\perp, \|}\left(\right.$ in $\mathrm{eV}^{-1}$ ) and $\gamma_{\perp, \|}($ in $\mathrm{eV}$ ):

for the case $\mathrm{D}_{1}(10)-$

$\beta_{\perp}=11.65, \gamma_{\perp}=8.03, \beta_{\|}=9.83, \quad \gamma_{\|}=4.28$;

for the case $\mathrm{D}_{2}(11)$

$\beta_{\perp}=14.20, \gamma_{\perp}=10.47, \beta_{\|}=7.22, \quad \gamma_{\|}=2.32$.

As it follows from the latter formulas, the components of absorption coefficient are bounded with the proportion $\alpha_{\|} / \alpha_{\perp}=k_{\|} / k_{\perp}=A_{\|}^{*} / A_{\perp}^{*}$. According to [26], the experimental ratio $k_{\|} / k_{\perp}$ for the case of the fundamental absorption in $\mathrm{CuInSe}_{2}$ crystals varies within the range 1.05-1.10. The same ratio, calculated according from (15) using (16) and (17), would yield the values 1.44 and 1.75 for the cases $D_{1}$ and $D_{2}$, respectively. Therefore, the best fit of theoretical curves to the experimental data regarding $\alpha_{\|} / \alpha_{\perp}$ ratio can be attained by using the hole effective masses according to the case $\mathrm{D}_{1}$ (formula (10)).

It was experimentally proven $[28,29]$ that in quasicubic model approximation for the crystals with chalcopyrite structure [30] illuminated by light wave with the polarization vector perpendicular to the main optical axis of the crystal, the ratio of intensity peak in electro-reflection spectra $\left(I_{\|}\right.$and $\left.I_{\perp}\right)$ obeys the following expressions:

$\frac{I_{\|}}{I_{\perp \mid A}}=0, \quad \frac{I_{\|}}{I_{\perp \mid B, C}}=9 \cdot\left(2 / 3-E_{\mp} / \Delta_{s o}\right)^{2}$

with

$$
E_{\mp}=0.5 \cdot\left(\Delta_{s o}+\Delta_{c r} \mp \sqrt{\left(\Delta_{s o}+\Delta_{c r}\right)^{2}-\frac{8}{3} \Delta_{s o} \Delta_{c r}}\right) \cdot
$$

The discussed peaks correspond to electron transitions from the valence to conduction band in the crystals $\mathrm{A}^{\mathrm{II}} \mathrm{B}^{\mathrm{IV}} \mathrm{C}^{\mathrm{V}}{ }_{2}$. It is worth noting that for the limit case $\Delta_{s o} \rightarrow 0$ the ratio $E_{-} / \Delta_{s o}$ tends to $2 / 3$. Taking into account (5), (6), one can show that for the crystals with $C_{1}>1$

$\frac{I_{\|}}{I_{\perp \mid C}}=2 \cdot C_{1}, \quad \frac{I_{\|}}{I_{\perp \mid B}}=2 / C_{1}$.

For the case $\mathrm{C}_{1}<1$, the subscripts " $\mathrm{B}$ " and " $\mathrm{C}$ " should be swapped in (20).

In this way, one can treat the ratio $\alpha_{\|} / \alpha_{\perp}=A_{\|} / A_{\perp}$ as a function of the parameter $C_{1}$ for the crystals with chalcopyrite or wurtzite structure. The inset to Fig. 4 proves that the dependence $\left(A_{\|}^{*} / A_{\perp}^{*}-A_{\|}^{*}(0) / A_{\perp}^{*}(0)\right) \cdot 100 \%$ is linear and equal to $(8 \pm 1) \cdot C_{1}$ or $(21 \pm 2) \cdot C_{1}$ for the hole masses calculated for the cases $\mathrm{D}_{1}$ and $\mathrm{D}_{2}$. Experimental data on this ratio for $\mathrm{p}$ $\mathrm{CuInSe}_{2}$ (shown as the asterisk in the inset to Fig. 4) fits better to the case when the effective mass estimations are done with the formula (10). The obtained correlations 
between $A_{\perp, \|}$ and their ratio for the different wurtzite and chalcopyrite crystals (e.g., $\mathrm{CuInSe}_{2}, \mathrm{CdSe}, \mathrm{CdS}$ and $\mathrm{ZnO}$, see Fig. 4) suggests that the most accurate description of the effective mass tensor for $\mathrm{p}-\mathrm{CuInSe}_{2}$ is that provided with the formulas (10).

\section{Conclusions}

We show that $\mathrm{p}-\mathrm{CuInSe} \mathrm{e}_{2}$ crystals with the components of hole effective mass tensor obeying the symmetry relations (3) is characterized with two sets of $m_{\perp, \|}^{A, B, C}$, describing temperature dependence of the total conductivity, Hall coefficient and thermal voltage within experimental precision. For the upper valence sub-bands $\Gamma_{6}^{-}$and $\Gamma_{7}^{+}$, we have estimated the coefficients $C_{A, B}$ characterizing deviation of the dispersion relation $E(\mathrm{k})$ from its parabolic form, which yielded an acceptable agreement of the calculated and experimental temperature dependences for the kinetic coefficients in this material. It was found that $C_{A, B}=k_{A, B}\left(E_{0}-E_{g}\right)$ for $\mathrm{p}-\mathrm{CuInSe} \mathrm{C}_{2}$ and their $\mathrm{A}^{\mathrm{II}} \mathrm{B}^{\mathrm{VI}}$ analogs, allowing to refine the values of $C_{A}$ for $\mathrm{CdSe}$ and $\mathrm{CdS}$. We also obtained a phenomenological expression (15) for the coefficients $A_{\perp, \|}$ in formula (13) describing the spectral dependence of light absorption tensor close to the fundamental absorption edge as a function of $E_{g}$. Moreover, the dependence $\left(A_{\|}^{*} / A_{\perp}^{*}-A_{\|}^{*}(0) / A_{\perp}^{*}(0)\right) \cdot 100 \%=f\left(C_{1}\right)$ proved to be linear in the first approximation for $\mathrm{CdSe}$, $\mathrm{CdS}, \mathrm{ZnO}$ and $\mathrm{CuInSe}_{2}$ with wurtzite and chalcopyrite structure; the experimental data known for $\mathrm{p}-\mathrm{CuInSe} \mathrm{Sits}_{2}$ reasonably our theoretical calculations performed for the components of the effective masses given by the formula (10).

The obtained effective mass values make it possible to properly explain the component anisotropy for the interband light absorption tensor, as well as the experimental data on temperature dependence of the kinetic coefficients within $\mathrm{p}-\mathrm{CuInSe} \mathrm{S}_{2}$ in the temperature range $100-350 \mathrm{~K}$.

\section{Acknowledgements}

The paper was partially supported by the budget financing of the Ministry for Education and Science of Ukraine and research projects (2009-2011 years) at the Department of Electronics and Energy Engineering and Scientific and Educational Center "Material Science of Semiconductors and Energy-Efficient Technologies" at the Chernivtsi National University.

\section{References}

1. H.W. Schock, Solar cells based on $\mathrm{CuInSe}_{2}$ and related compounds: recent progress in Europe // Sol. Energy Mater. 34, p. 19-26 (1994).
2. U. Rau, H.W. Schock, Electronic properties of $\mathrm{Cu}(\mathrm{In}, \mathrm{Ga}) \mathrm{Se}_{2}$ heterojunction solar cells-recent achievements, current understanding, and future challenges // Appl. Phys. A 69, p. 131-147 (1999).

3. J.E. Jaffe, A. Zunger, Electronic structure of the ternary chalcopyrite semiconductors // Phys. Rev B 28, p. 5822-5846 (1983).

4. J.E. Jaffe, A. Zunger, Theory of band-gap anomaly in $\mathrm{ABC}_{2}$ chalcopyrite semiconductors // Phys. Rev. $B$ 29, p. 1882-1906 (1984).

5. H.T. Shaban, M. Mobarak, M.M. Nassary, Characterization of $\mathrm{CuInSe}_{2}$ single crystal // Physica B 389, p. 351-354 (2007).

6. V.A. Chaldyshev, G.F. Karavaev, To a question about energy spectra structure in chalcopyrite crystals // News of the High Education Institutions 2, p. 28-30 (1964).

7. G.F. Karavaev, A.S. Poplavnoy, Investigation of the energy spectra for the electrons in semiconductor compounds with chalcopyrite lattice using a perturbation theory // Fizika tverdogo tela 8, p. 2144-2148 (1966) (in Russian).

8. H. Neumann, W. Kissinger, H. Sobotta, V. Riede, G. Kühn, Hole effective masses in CuInSe 2 // Phys. status solidi (b) 108 (2), p. 483-487 (2006).

9. P.M. Gorley, O.O. Galochkina, Yu.V. Vorobiev, J. González-Hernández, Temperature dependence of kinetic coefficients and thermal voltage for $\mathrm{p}$ $\mathrm{CuInSe}_{2}$ crystals // Thermoelectric 2, p. 48-56 (2008).

10. P.P. Horley, V.V. Gorley, P.M. Gorley, J. GonzalezHernandez, Yu.V. Vorobiev, On correlation of CdS and CdSe valence band parameters // Thin Solid Films 480-481, p. 373-376 (2005).

11. E. Gutsche, E. Jahne, Spin-orbit splitting of the valence band of wurtzite type crystals // Phys. status solidi (b) 19, p. 823-832 (1967).

12. E. Jahne, E. Gutsche, Valence band structure of wurtzite type crystals // Phys. status solidi 21, p. 57-68 (1967).

13. K. Yoodee, J.C. Woolley, V. Sa-yakanit, Effects of p-d hybridization on the valence band of I-III- $-\mathrm{VI}_{2}$ chalcopyrite semiconductors // Phys. Rev. B 30 (10), p. 5904-5915 (1984).

14. W.R.L. Lambrecht, A.V. Rodina, S. Limpijumnong, D. Segall, B.K. Meyer, Valenceband ordering and magneto-optic exciton fine structure in $\mathrm{ZnO} / /$ Phys. Rev. B 65, 075207-1075207-12 (2002).

15. L.C. Lew Yan Voon, M. Willatzen, M. Cardona, N.E. Christencen, Terms linear in $\mathrm{k}$ in the band structure of wurtzite-type semiconductors // Phys. Rev. B 53(16), p. 10703-10714 (1996).

16. E.I. Rashba, Symmetry of the energy bands in wurtzite crystals // Fizika tverdogo tela 1(3), p. 407-421 (1959) (in Russian).

17. M. Cardona, Band parameters of semiconductors with zincblende, wurtzite, and germanium structure // J. Phys. Chem. Solids 24, p. 1543-1555 (1963). 
18. J.L. Shay, B. Tell, H.M. Kasper, L.M. Schiavone, Electronic structure of $\mathrm{AgInSe}_{2}$ and $\mathrm{CuInSe}_{2} / /$ Phys. Rev. B 7, p. 4485-4490 (1973).

19. A. Continenza, R.M. Wentzcovitch, A.J. Freeman, Theoretical investigation of graphitic $\mathrm{BeO} / /$ Phys. Rev. B 41(6), p. 3540-3544 (1990).

20. T.S. Moss, G.J. Burrell, B. Ellis, Semiconductor Opto-Electronics. Mir Publ., Moscow, 1976 (in Russian).

21. P.M. Gorley, V.V. Khomyak, Yu.V. Vorobiev, J. Gonzalez-Hernandez, P.P. Horley, O.O. Galochkina, Electron properties of n- and p$\mathrm{CuInSe}_{2}$ // Solar Energy 82, p. 100-105 (2008).

22. C. Rincon, R. Marquez, Defect physics of the $\mathrm{CuInSe}_{2}$ chalcopyrite semiconductors // J. Phys. Chem. Solids 60, p. 1865-1873 (1999).

23. H. Neumann, R.D. Tomlinson, Band-gap narrowing in n-type CuInSe $\mathrm{S}_{2}$ single crystals // Solid State Communs 57, p. 591-594 (1986).

24. I. Hernandez-Calderon, Optical properties and electronic structure of wide band gap II-VI semiconductors, Chap. 4, In: II-VI Semiconductor
Materials and their Applications, Eds. M.C. Tamargo. Taylor and Francis, New York, 2002, p. 113-170.

25. J.D. Park, B.H. Chang, I.H. Choi, Thermoelectric power of p-CuInSe ${ }_{2}$ single crystals // J. Korean Phys. Soc. 22, p. 113-213 (1989).

26. M.I. Alonso, K. Wakita, J. Pascual, M.Garriga, N. Yamamoto, Optical functions and electronic structure of $\mathrm{CuInSe}_{2}, \mathrm{CuGaSe}_{2}, \mathrm{CuInS}_{2}$ and $\mathrm{CuGaS}_{2}$ // Phys. Rev. B 63, 075203-1-075203-13 (2001).

27. T. Irie, S. Endo, S. Kimura, Electrical properties of p- and n-type CuInSe $\mathrm{C}_{2}$ single crystals // Jpn J. Appl. Phys. 18, p. 1303-1310 (1979).

28. O. Madelung, U. Rössler, M. Schulz, Zinc oxide ( $\mathrm{ZnO})$ electron effective masses, 41B, In: II-VI and I-VII Compounds; Semimagnetic Compounds. Springer-Verlag, 2006.

29. J.L. Shay, E. Buehler, Electroreflectance study of the energy-band structure of $\mathrm{CdSnP}_{2} / /$ Phys. Rev. B 2(10), p. 4104-4109 (1970).

30. J.E. Rowe, J.L. Shay, Extension of the quasi-cubic model to ternary chalcopyrite crystals // Phys. Rev. B 3(2), p. 451-453 (1971). 